\title{
CONTRASTES DE GÉNERO EN UNA EXPERIENCIA DE FORMACIÓN E-LEARNING EN EL GRADO DE MAESTRO DE LA FACULTAD DE EDUCACIÓN DE ALBACETE
}

\author{
GENDER CONTRASTS IN AN E-LEARNING TRAINING EXPERIENCE IN THE \\ PRIMARY TEACHER DEGREE OF THE FACULTY OF EDUCATION OF ALBACETE \\ CONTRASTES DE GÊNERO EM UMA EXPERIÊNCIA DE FORMAÇÃO E-LEARNING \\ NO CURSO DE PROFESSOR PRIMÁRIO DA FACULDADE DE EDUCAÇÃO DE \\ ALBACETE
}

\author{
Ascensión Palomares-Ruiz \\ Universidad De Castilla-La Mancha, Espanha \\ ascension.palomares@uclm.es \\ Antonio Cebrián \\ Universidad De Castilla-La Mancha, Espanha \\ antonio.cebrian@uclm.es \\ Emilio López-Parra \\ Universidad De Castilla-La Mancha, Espanha \\ emilio.lopezparra@uclm.es
}

\begin{abstract}
RESUMEN: El E-learning, electronic learning, o aprendizaje online, se refiere al aprendizaje mediante internet y la tecnología siendo sus principales beneficios la eliminación de las barreras espaciotemporales y la posibilidad de acceder al "lifelong learning" o el aprendizaje permanente a lo largo de la vida adaptado a las necesidades personales siendo una modalidad formativa que pretende aportar flexibilidad e individualización en los procesos de enseñanza-aprendizaje. El objetivo de la investigación fue verificar, mediante el análisis estadístico inferencial, si es posible rechazar la hipótesis nula de igualdad de género en las medias muestrales de las calificaciones como medición del rendimiento académico entre una materia de segundo del Grado de Maestro de Primaria de la Facultad de Educación de Albacete impartida con una metodología centrada en la formación E-learning con herramientas TIC de la Web 3.0 como Padlet, Socrative y Kahoot y otra materia impartida con una metodología más tradicional basada únicamente en las herramientas proporcionadas por el campus virtual. Se recurrió a un método cuasiexperimental a través de grupo de control no equivalente y no se encontraron diferencias de género estadísticamente significativas. Los resultados concuerdan con los datos proporcionados por Eurostat que constatan una brecha digital de género que está relacionada con la baja matriculación de mujeres en carreras técnicas y deberán alentar al profesorado a la inclusión, en la virtualización de sus materias, de variadas herramientas TIC que posibiliten la transición hacia metodologías más participativas y colaborativas con una mayor implicación y compromiso del alumnado.
\end{abstract}

PALABRAS CLAVE: E-learning. Brecha digital de género. Aprendizaje en red. Aprendizaje colaborativo.

ABSTRACT: E-learning, electronic learning refers to learning through the internet and technology, its main benefits being the elimination of space-time barriers and the 
possibility of accessing "lifelong learning" or lifelong learning throughout the life adapted to personal needs, being a training modality that aims to provide flexibility and individualization in the teaching-learning processes. The objective of the research was to verify, by means of inferential statistical analysis, whether it is possible to reject the null hypothesis of gender equality in the sample means of the qualifications as a measure of academic performance between a subject of second course of the Primary Teacher Degree of the Albacete Faculty of Education taught with a methodology focused on Elearning with Web 3.0 ICT tools such as Padlet, Socrative and Kahoot and another subject taught with a more traditional methodology based solely on the tools supplied by the virtual campus. A quasi-experimental method with a non-equivalent control group was used and no statistically significant gender differences were found. The results are in agreement with the data provided by Eurostat that confirm a digital gender gap that is related to the low enrollment of women in technical careers and should encourage teachers to include, in the virtualization of their subjects, various ICT tools that enable the transition towards more participatory and collaborative methodologies with greater involvement and commitment of the students.

KEYWORDS: E-learning. Digital gender gap. Network learning. Collaborative learning.

RESUMO: E-learning, aprendizagem electrônica ou aprendizagem online refere-se à aprendizagem por meio da Internet e da tecnologia, tendo como principais benefícios a eliminação das barreiras espaço-temporais e a possibilidade de acesso à "aprendizagem ao longo da vida" ou aprendizagem permanente ao longo do vida adaptada às necessidades pessoais, sendo uma modalidade de formação que visa proporcionar flexibilidade e individualização nos processos de ensino-aprendizagem. O objetivo da pesquisa foi verificar, por meio de análise estatística inferencial, se é possível rejeitar a hipótese nula de igualdade de gênero nas médias amostrais das notas como medida de desempenho acadêmico de uma segunda disciplina do Curso de Licenciatura do Ensino Fundamental. A Faculdade de Educação de Albacete lecionava com uma metodologia voltada para o treinamento E-learning com ferramentas TIC da Web 3.0, como Padlet, Socrative e Kahoot, e outra disciplina ministrada com uma metodologia mais tradicional baseada exclusivamente nas ferramentas disponibilizadas pelo campus virtual. Um método quase experimental foi usado por meio de um grupo de controle não equivalente e não foram encontradas diferenças de gênero estatisticamente significativas. Os resultados são consistentes com os dados fornecidos pelo Eurostat que confirmam um fosso digital de gênero relacionado com a baixa inscrição das mulheres nas carreiras técnicas e devem encorajar os professores a incluir, na virtualização das suas disciplinas, várias ferramentas TIC que possibilitem a transição para metodologias mais participativas e colaborativas com maior envolvimento e empenho dos alunos.

PALAVRAS-CHAVE: E-learning. Diferença digital de gênero. Aprendizagem em rede. Aprendizagem colaborativa.

\section{Introducción}

Numerosos estudios en torno a diferencias culturalmente establecidas entre géneros reflejan que se promueve menos a las mujeres hacia las carreras técnicas (ADYA; KAISER, 2005; CASTAÑO, 2008; ESPEJO, 2015; FERNÁNDEZ et al., 2008; 
FERNÁNDEZ; FERNÁNDEZ; RODRÍGUEZ, 2018; MATEOS; GÓMEZ, 2019; SÁINZ; LÓPEZ-SÁEZ, 2010), señalando que, en las dos últimas décadas, desciende su presencia (BURRELLI, 2008; CARLSON, 2006; GONZÁLEZ; VERGÉS; MARTíNEZ, 2017). Además, frecuentemente, la tecnología y los ordenadores son considerados socialmente como herramientas masculinas (ADYA; KAYSER, 2005; GLEDHILL, 2019; JENSON; CASTELL, 2010; ROYSE et al., 2007; SÁINZ; LÓPEZ-SÁEZ, 2010). En esta línea, entre los factores implicados en dicho rechazo, destacan que las mujeres hacen un menor uso de los videojuegos, siendo estos una puerta de entrada al mundo de la tecnología (BEAVIS; CHARLES, 2007; GIL-JUÁREZ; FELIU; GONZÁLEZ, 2010; JENSON; CASTELL, 2010; RUIZ; SÁNCHEZ, 2010).

Las investigaciones apuntan a la existencia de tres brechas digitales de género, la primera se revela en el acceso a la tecnología y es de carácter cuantitativo, la segunda se manifiesta en el uso que se hace de la tecnología y es de carácter cualitativo, y la tercera está relacionada con la utilización de los servicios TIC más avanzados y es también de carácter cualitativo (CASTAÑO, 2008; GARCÍA-VALCÁRCEL; TEJEDOR, 2017). La primera brecha digital de género se está reduciendo gracias al mayor uso de la tecnología; de hecho, en muchos países las mujeres son usuarias de las TIC en cifras más parecidas cada vez, si no superiores, a las de los hombres (BOOTH; GOODMAN; KIRKUP, 2010; BRYNIN, 2006; CHAVES; TRUJILLO; LÓPEZ, 2015; MATEOS; GÓMEZ, 2019). Sin embargo, la segunda y tercera brecha digital de género, trascienden al simple acceso y uso de las TIC y al despliegue de habilidades navegadoras básicas 0 informáticas, estando vinculadas con la baja representación de las mujeres en sectores estratégicos de la tecnología (BOOTH; GOODMAN; KIRKUP, 2010; CASTAÑO, 2008; FERNÁNDEZ; FERNÁNDEZ; RODRÍGUEZ, 2018)

Los datos proporcionados por Eurostat (2019a) referidos a la frecuencia de acceso a internet menos de una vez a la semana sobre el porcentaje de personas que usaron internet en los últimos 3 meses, constatan que la primera brecha digital de género se está reduciendo (Ver Tabla 1 y la Figura 1), coincidiendo con lo señalado en las investigaciones internacionales.

Tabla 1: Frecuencia de acceso a internet.

\begin{tabular}{|l|c|c|c|c|c|c|c|c|c|c|c|c|c|c|}
\hline GEO/TIME & 2005 & 2006 & 2007 & 2008 & 2009 & 2010 & 2011 & 2012 & 2013 & 2014 & 2015 & 2016 & 2017 & 2018 \\
\hline $\begin{array}{l}\text { EU - Males,16 to } \\
74 \text { years old }\end{array}$ & 12 & 11 & 9 & 8 & 6 & 4 & 5 & 4 & 4 & 3 & 3 & 3 & 3 & 2 \\
\hline $\begin{array}{l}\text { EU -Females,16 } \\
\text { to 74 years old }\end{array}$ & 19 & 15 & 13 & 11 & 8 & 7 & 6 & 6 & 5 & 5 & 4 & 4 & 3 & 2 \\
\hline $\begin{array}{l}\text { Spain-Males,16 } \\
\text { to 74 years old }\end{array}$ & 18 & 15 & 13 & 12 & 9 & 8 & 8 & 6 & 6 & 6 & 5 & 5 & 6 & 4 \\
\hline $\begin{array}{l}\text { Spain- } \\
\begin{array}{l}\text { Females,16 to } \\
74 \text { years old }\end{array}\end{array} 25$ & 21 & 17 & 16 & 13 & 10 & 9 & 9 & 10 & 7 & 5 & 5 & 5 & 4 \\
\hline
\end{tabular}

Fuente: Elaboración propia adaptado de Eurostat (2019a). 
Figura 1: Frecuencia de acceso a internet.

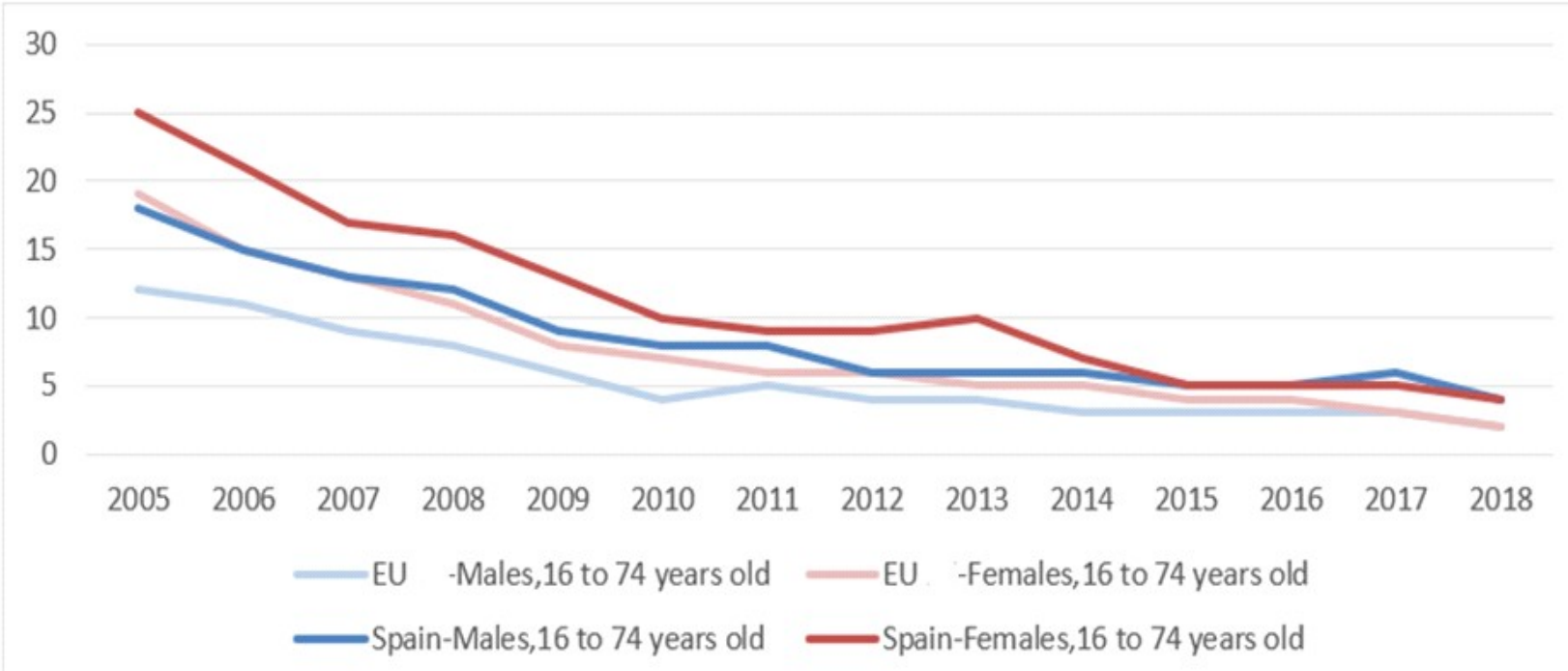

Fuente: Elaboración propia adaptado de Eurostat (2019a).

La segunda y tercera brecha digital de género persisten en el tiempo y están relacionadas con la baja matriculación de mujeres en carreras técnicas, como puede apreciarse en las Tablas 2 y 3, y en la Figura 2, con datos de Eurostat (2019b) respecto a la distribución del alunado de nuevo ingreso a los estudios de Grado por ámbito de estudio y sexo. Se puede observar que, las mujeres se decantan por carreras relacionadas con la educación, la salud y el sector servicios, mientras que los hombres se inclinan por las carreras técnicas, ingenierías e informática, estacando que en estos estudios las mujeres apenas llegan al 10\%.

Tabla 2: Estudiantes matriculados en educación terciaria

\begin{tabular}{|l|c|c|c|c|c|c|c|c|c|c|c|}
\hline GEO/TIME & \multicolumn{2}{|c|}{2013} & \multicolumn{2}{|c|}{2014} & \multicolumn{2}{|c|}{2015} & \multicolumn{2}{|c|}{2016} & \multicolumn{2}{c|}{2017} \\
\hline European Union-28 countries & Males\% & $\begin{array}{c}\text { Females } \\
\%\end{array}$ & $\begin{array}{c}\text { Males } \\
\%\end{array}$ & $\begin{array}{c}\text { Females } \\
\%\end{array}$ & $\begin{array}{c}\text { Males } \\
\%\end{array}$ & $\begin{array}{c}\text { Females } \\
\%\end{array}$ & $\begin{array}{c}\text { Males } \\
\%\end{array}$ & $\begin{array}{c}\text { Females } \\
\%\end{array}$ & $\begin{array}{c}\text { Males } \\
\%\end{array}$ & $\begin{array}{c}\text { Females } \\
\%\end{array}$ \\
\hline Education & 21,80 & 78,24 & 22,23 & 77,84 & 21,34 & 78,72 & 21,63 & 78,41 & 21,94 & 78,10 \\
\hline Health and Welfare & 27,73 & 72,32 & 27,90 & 72,10 & 28,82 & 71,24 & 28,91 & 71,11 & 28,74 & 71,33 \\
\hline Arts and Humanities & 34,84 & 65,23 & 35,34 & 64,71 & 35,10 & 64,90 & 35,01 & 64,90 & 35,50 & 64,52 \\
\hline $\begin{array}{l}\text { Social sciences, Journalism and } \\
\text { Information }\end{array}$ & 37,54 & 62,52 & 37,54 & 62,52 & 36,81 & 63,24 & 36,01 & 64,02 & 36,01 & 63,93 \\
\hline $\begin{array}{l}\text { Business, Administration and Law } \\
\text { Agriculture, Forestry, Fisheries }\end{array}$ & 43,94 & 56,14 & 44,71 & 55,31 & 44,31 & 55,72 & 44,50 & 55,51 & 45,51 & 54,50 \\
\hline $\begin{array}{l}\text { Nand Veterinary } \\
\text { and Statistics }\end{array}$ & 50,33 & 49,74 & 49,94 & 50,13 & 49,80 & 50,21 & 49,80 & 50,24 & 49,47 & 50,34 \\
\hline Services & & & 82,41 & 17,62 & 52,74 & 47,32 & 52,73 & 47,34 & 52,84 & 47,23 \\
\hline $\begin{array}{l}\text { Engineering, Manufacturing and } \\
\text { Construction }\end{array}$ & & & & 73,83 & 26,20 & 74,02 & 26,01 & 73,92 & 26,13 \\
\hline $\begin{array}{l}\text { Information and Communication } \\
\text { Technologies }\end{array}$ & 82,63 & 17,34 & 82,41 & 17,64 & 81,93 & 18,10 & 81,72 & 18,30 & 82,02 & 18,03 \\
\hline
\end{tabular}

Fuente: Elaboración propia adaptado de Eurostat (2019b). 
Belo Horizonte, v. 13, n. 3, p. 161-180, set.-dez. 2020 - ISSN 1983-3652 DOI: $10.35699 / 1983-3652.2020 .25114$

Figura 2: Estudiantes matriculados en educación terciaria.

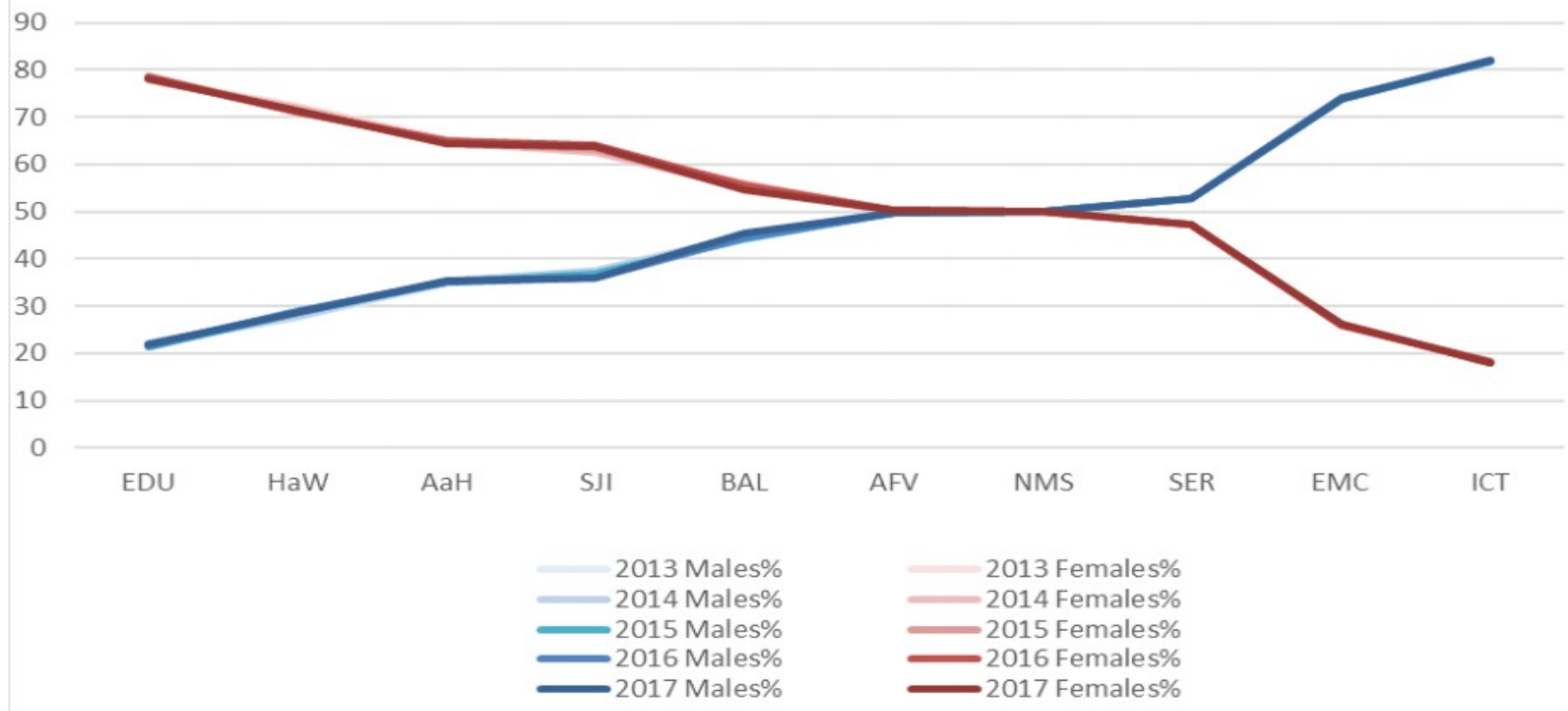

Fuente: Elaboración propia adaptado de Eurostat (2019b).

Tabla 3: Ámbitos de estudio y acrónimos utilizados en la Figura 2.

\begin{tabular}{|c|l|}
\hline EDU & Education \\
\hline HaW & Health and Welfare \\
\hline AaH & Arts and Humanities \\
\hline SJI & Social sciences, Journalism and Information \\
\hline BAL & Business, Administration and Law \\
\hline AFV & Agriculture, Forestry, Fisheries and Veterinary \\
\hline NMS & Natural sciences, Mathematics and Statistics \\
\hline SER & Services \\
\hline EMC & Engineering, Manufacturing and Construction \\
\hline ICT & Information and Communication Technologies \\
\hline
\end{tabular}

Fuente: Elaboración propia adaptado de Eurostat (2019b).

El e-learning es el acrónimo en inglés abreviado de electronic learning, refiriéndose a la enseñanza y aprendizaje online, mediante Internet y la tecnología. También conocido como formación online, enseñanza virtual, teleformación o formación a distancia. Actualmente, Internet es el mejor canal de acceso a todo tipo de formación online, siendo uno de los principales beneficios que proporciona la eliminación de las barreras físicotemporales, de tiempo, así como posibilitar el "lifelong learning", o aprendizaje a lo largo de la vida, permitiendo adaptarlo a las necesidades personales de cada alumno, aportando personalización y flexibilidad en los procesos de enseñanza-aprendizaje (BARTOLOMÉ, GARCÍA-RUIZ; AGUADED, 2018; GARCÍA, 2005).

Masie (2005), acuñó el término e-learning, elaborando una definición que todavía está actualizada, especificando que implica el uso de internet, e incluye la tecnología móvil y todo uso de la tecnología que tienda a expandir, reforzar, distribuir, desarrollar, evaluar, certificar o acelerar los procesos de aprendizaje, abarcando elementos tales como gestión del conocimiento, colaboración y apoyo al ejercicio.

Resulta importante subrayar que, Lozano (2004) formuló, en el año 2002, la teoría del "Triángulo del e-learning" destacando los tres pilares esenciales (tecnología, contenidos y acción docente) que debe tener cualquier proyecto de e-learning, estando 
todos interconectados entre sí, y careciendo de sentido los unos sin los otros. La tecnología estaría relacionada con la plataforma o entorno de aprendizaje virtual llamado Learning Management System (LMS), donde se desarrolla el curso y se gestionan los contenidos, manteniendo la relación de comunicación entre los alumnos y los docentes, como el Moodle de código abierto (opensource) que está bajo la concreción tecnológica de los servicios de campus virtual de muchas universidades, que reproducen las prácticas y metodologías de las clases tradicionales en formato virtual, obteniendo unos logros similares (ADELL; CASTAÑEDA, 2012). Pensamos que se están creando las condiciones adecuadas para que se produzca una revolución en un mundo en transición, desde la sociedad de la información, hacia la sociedad del conocimiento. No obstante, para que esta innovación pueda arribar a las aulas y, por ende, al sistema educativo, es necesario que se supere la etapa de integración de las tecnologías emergentes con los sistemas educativos tradicionales, y han de florecer unas pedagogías emergentes, sustentadas en teorías como el cognitivismo, el constructivismo, el aprendizaje significativo, y otros nuevos planteamientos desarrollados tras el desarrollo de la web 3.0 y de Internet, como el aprendizaje autorregulado, el aprendizaje rizomático, la teoría LaaN, el conectivismo y el aprendizaje entre iguales, que no han acabado de impregnar los usos educativas tradicionales. En este contexto, de incorporación y correcto empleo de las TIC, se están creando las condiciones adecuadas para que con estas concepciones pedagógicas nuevas y viejas eclosionen y estimulen una auténtica transformación de la docencia, poniendo el foco de atención en el aprendizaje en vez de en la enseñanza, en el alumnado en vez de los profesores, permitiendo que deje de ser un sujeto pasivo y se convierta en un sujeto activo de su propio aprendizaje (AREA; ADELL, 2009; BADIA; MENESES; SIGALÉS, 2013; CABERO, 2006).

La investigación que presentamos se realizó en diferentes grupos de alumnos de segundo curso del Grado de Maestro en Educación Primaria de la materia Educación y Sociedad, en idioma inglés: Education and Society (en adelante EyS), en la Facultad de Educación de Albacete en la Universidad de Castilla La Mancha (UCLM), durante el curso 2018-19. El objetivo de la investigación fue el verificar, a través el análisis estadístico inferencial, si podía ser refutada la hipótesis nula de la igualdad de las medias muestrales de las calificaciones como medición del rendimiento académico entre la materia EyS impartida en idioma inglés mediante una metodología que hacía uso de las herramientas habituales del campus virtual añadiendo otras herramientas TIC de la Web 3.0, como Socrative y Padlet, y la misma materia impartida en idioma castellano en otros grupos de la Facultad por otros profesores distintos del Departamento de Pedagogía, mediante una metodología más tradicional centrada únicamente en las herramientas suministradas por el campus virtual, y que configuraron el grupo de control, también se verificó la existencia de diferencias de género estadísticamente significativas que nos permitiesen hablar de la existencia de una brecha digital de género.

\section{Método}

En la investigación, se elaboró un contraste cuantitativo de resultados de aprendizaje y contrastamos, a través del análisis estadístico inferencial, la hipótesis de investigación: ¿La utilización en el campus virtual de herramientas TIC de la Web 3.0, como Socrative y Padlet, favorecían el rendimiento académico del alumnado de segundo 
curso del Grado de Maestro de Primaria en la Facultad de Educación de Albacete, respecto al uso en exclusiva de las herramientas tradicionales del campus virtual? Además, se verificó si existían diferencias de género estadísticamente significativas que permitan poder afirmar el haber encontrado una brecha digital de género entre el alumnado. Igualmente, se analizaron algunas de las ventajas e inconvenientes del empleo de las nuevas tecnologías en la educación universitaria frente a métodos más tradicionales centrados en el manejo único de las herramientas del campus virtual.

Dentro de un enfoque cuantitativo utilizamos un método cuasiexperimental, porque lo que se pretendía era establecer correlaciones causales entre las variables involucradas, tratando de esclarecer hasta qué punto los variaciones observados en las variables dependientes son fruto de la manipulación realizada sobre la variable independiente, valiéndonos de la estadística inferencial. Se pretendía que la investigación se desarrollase en un contexto lo más "ecológico" posible; para lo cual se requería que las circunstancias en las que se desarrollase el estudio fuesen análogas a las que se podían hallar en la docencia universitaria usual de la Facultad de Educación de Albacete, donde fue llevada a cabo la investigación. Por ello, se seleccionaron grupos ya formados, sin asignar aleatoriamente al alumnado. Siendo conscientes que esta elección reduce la validez externa de la investigación, por lo que no podemos afirmar que los resultados obtenidos fuesen generalizables, no siendo, por tanto, grupos equivalentes. Estas condiciones no fueron determinantes, puesto que el objetivo de la investigación no fue el generalizar los resultados, sino tratar de comprender las relaciones que pudiesen existir entre las variables objeto del análisis. Otro aspecto que tratamos de controlar fue la posible influencia del profesor en los grupos de control y experimental, al resultar imposible que la docencia en ambos grupo fuese impartida por el mismo profesor, tratamos de minimizar la influencia de esta variable al ser todos los profesores del mismo departamento el de Pedagogía.

\subsection{Participantes}

Se tomó como muestra en la investigación la población total de alumnos de segundo curso del Grado de Maestro de Educación Primaria de las materias Educación y Sociedad, en el curso 2018-2019, con un tamaño de 121 participantes. El alumnado ya estaba asignado en 3 grupos diferentes, dos del turno de mañana y un grupo del turno de tarde, con anterioridad a la investigación. Lógicamente, por lo que no se pudieron asignar aleatoriamente $y$, por tanto, no fueron grupos equivalentes. Se seleccionó el grupo B como el grupo experimental y, el grupo C como el grupo de control, mientras que el grupo A no participó en la investigación. Estas condiciones no fueron determinantes en la investigación porque el objetivo era el tratar de comprender la existencia de posibles relaciones entre las variables del estudio. En la Tabla 4 puede observarse que la muestra quedó constituida por 62 alumnos, 37 dentro del grupo de control y 25 dentro del grupo experimental.

Tabla 4: Población y muestras.

\begin{tabular}{|l|c|c|c|c|}
\hline \multicolumn{1}{|c|}{ Grupo } & Curso & Población & Muestra & TOTAL \\
\hline Experimental & 2B & 25 & 25 & \multirow{2}{*}{62} \\
\hline Control & 2C & 42 & 37 & \\
\hline No participa & 2A & 54 & & 54 \\
\hline
\end{tabular}


Belo Horizonte, v. 13, n. 3, p. 161-180, set.-dez. 2020 - ISSN 1983-3652

DOI : $10.35699 / 1983-3652.2020 .25114$

\begin{tabular}{|l|c|c|c|c|}
\hline TOTALES & & 121 & 62 & 116 \\
\hline \multicolumn{4}{|c|}{ Fuente: Elaboración própria. }
\end{tabular}

\subsection{Procedimiento}

La investigación fue realizada con una metodología cuasiexperimental a través de grupo de control no equivalente. Para el análisis estadístico se manejaron 2 programas de análisis de datos gratuitos y de código abierto que existen gracias al trabajo de un sinfín de desarrolladores en todo el mundo como PSPP y R implementando el paquete Rkward.

Se contemplaron las siguientes variables:

- La variable independiente cualitativa dicotómica fue la metodología de trabajo que tomó dos valores: S (utilización en el campus virtual de herramientas TIC de la Web 3.0 como Socrative y Padlet) y $\mathrm{N}$ (empleo único de las herramientas del campus virtual).

- Otra variable independiente dicotómica que estudió en la investigación fue el Género (GEN) que tomó dos valores: mujer (M) y hombre $(H)$.

- Las variables dependientes cuantitativas continuas fueron el rendimiento académico del alumnado, medido mediante las calificaciones en las actas ordinarias de la materia Eys dentro del grupo de $2^{\circ} \mathrm{B}$ que constituyó el grupo experimental, y dentro del grupo $2^{\circ} \mathrm{C}$ que constituyó el grupo de control.

Otras variables extrañas que podrían perjudicar los resultados de la investigación quedaron minimizadas por el diseño elegido. La diferencia fundamental entre la docencia en las materias en los grupos de control y experimental sería la utilización de herramientas TIC de la Web 3.0 como Socrative y Padlet en el campus virtual, dentro del grupo experimental 2B; mientras que dentro del grupo de control 2C, se emplearía únicamente las herramientas del campus virtual.

Dentro del grupo experimental se valoraron para el cálculo de la calificación final: las presentaciones y trabajos de los alumnos subidos al Padlet correspondiente (30\%), la elaboración de manera individual de un Portfolio Digital (20\%), la asistencia y participación en clase (10\%), y una prueba final tipo test de 100 preguntas: 45 de opción múltiple y 55 de verdadero/falso empleando la herramienta Socrative.

\subsection{Estudio de la fiabilidad de la prueba}

Para valorar la fiabilidad de la prueba; en el sentido de exactitud, estabilidad y precisión de los datos se escogió la fórmula Alfa de Cronbach, cuyo cálculo se efectuó de los datos obtenidos al realizar el cuestionario dentro de la muestra seleccionada. Como puede apreciarse en la Tabla 5, el resultado .669, al estar próximo a .70 se puede valorar que se obtiene una fiabilidad alta. Consecuentemente, se puede asumir la prueba como consistente internamente. También, se examinó la homogeneidad de los ítems, probando la correlación elemento-total y, se determinó que la eliminación de ninguno mejoraba substancialmente el Alfa de Cronbach. La prueba se efectuó en una de las salas de ordenadores de la Facultad de Educación de Albacete, mediante la herramienta Socrative. 
Belo Horizonte, v. 13, n. 3, p. 161-180, set.-dez. 2020 - ISSN 1983-3652 DOI : $10.35699 / 1983-3652.2020 .25114$

Tabla 5: Alfa de Cronbach en la prueba del grupo experimental EyS.

\begin{tabular}{|c|c|c|}
\hline \multicolumn{3}{|c|}{ Extracto del procesamiento de casos } \\
\hline Casos & $\mathrm{N}$ & $\%$ \\
\hline Válidos & 25 & 100.0 \\
\hline Excluidos & 0 & .0 \\
\hline Total & 25 & 100.0 \\
\hline \multicolumn{2}{|c|}{ Estadísticos de fiabilidad } \\
\hline Alfa de Cronbach & N de elementos \\
\hline .669 & 100 \\
\hline
\end{tabular}

Fuente: Elaboración própria.

\subsection{Recolección de datos}

Se consideró oportuno que, la única diferencia entre el grupo experimental y de control fuese la utilización de herramientas TIC de la Web 3.0, como Socrative y Padlet en el campus virtual, dentro del grupo experimental $2^{\circ} \mathrm{B}$, frente al empleo único de las herramientas tradicionales del campus virtual dentro del grupo de control $2^{\circ} \mathrm{C}$. El objetivo fundamental fue comparar las dos metodologías de trabajo. Una de estas metodologías, la más tradicional, estuvo centrada únicamente en las herramientas suministradas por el campus virtual; la otra metodología, empleando también de dicho campus virtual, no se limitó al empleo único de las herramientas suministradas por el mismo, sino que se incluyeron otras herramientas TIC de la Web 3.0, tales como Padlet y videos de repositorios como Vimeo o Youtube, que permitieron la creación de posters o murales digitales que incorporaron textos, imágenes, fotografías, vídeos, audios, dibujos, hipervínculos, etc. Además, de estas herramientas de la Web 3.0, se agregaron otras como Socrative empleada habitualmente para realizar presentaciones y poder sintetizar conceptos, para la realización de tormentas de ideas, la realización de ejemplos o resúmenes, pudiendo ser usado como un sistema de respuesta inteligente con el que el docente puede efectuar preguntas, quizzes y juegos a los que el alumnado puede responder en tiempo real desde sus propios dispositivos, ya sea desde un ordenador portátil, una Tablet o un móvil. Para aprovechar todas las posibilidades se organizaron distintos muros o padlets desde donde los estudiantes podrían bajarse los documentos dispuestos para cada tema, pudiendo también subir sus documentos de trabajo y presentaciones, tanto individuales como grupales (CATASÚS; ROMEU; PÉREZ-MATEO, 2007).

Se procuró utilizar una metodología activa, apoyada en los principios pedagógicos de: trabajo y autónomo y colaborativo-cooperativo, reflexión crítica a través de intercambios de ideas, debates y discusiones de diversas cuestiones, también se trabajó una metodología centrada en la resolución de problemas procurando que los propios alumnos resolvieran los problemas y explicasen los procesos de resolución a sus compañeros manejando un lenguaje apropiado, y animando en todo momento a los alumnos a descubrir y discutir diferentes procedimientos de resolución, pasando el papel del docente a ser el de guiar, animar, orientar, y motivar al alumnado, usando en la medida de lo posible recursos e instrumentos manipulativos, y empleando tanto materiales reales como virtuales, y teniendo en cuenta la atención a la diversidad con actividades de refuerzo y ampliación.

Se creó un Padlet de recursos TIC y otro para los enlaces a los Portfolios Digitales 
individuales, así como un Padlet para cada uno de los temas en los que se fraccionó la materia. En dichos Padlet se dispusieron los documentos de trabajo de los alumnos, con enlaces a videos y webs relacionadas con cada tema. Además, los alumnos también tuvieron que subir a los Padlet de cada tema sus trabajos y presentaciones grupales.

Cada alumno tuvo que desarrollar de manera personal un Portfolio Digital en el cual guardó todos sus trabajos y producciones teniendo que incluir sus reflexiones personales sobre lo estudiado en cada tema, así como sobre los recursos empleados, etc. igualmente, cada alumno tuvo que completar su rúbrica de evaluación e incluirla en su Portfolio. También se empleó la corrección por pares, según la cual un compañero de clase tuvo que verificar que todos los enlaces funcionaban y tuvo que completar la rúbrica de evaluación de otro Portfolio digital, además de la del suyo propio.

El Portfolio final tenía que ser un enlace a una red pública (donde no fuese necesario la instalación de ninguna aplicación ni recurso, ni el registro o la recepción de ninguna invitación) que incluía los enlaces o links a los recursos externos y propios seleccionados por cada alumno.

Cada alumno, tuvo que elegir una plataforma determinada donde se sintiese más cómodo para albergar su Portfolio digital (Blog, Wiki, Wix, Evernote, etc.), también se incluyeron ejemplos de Porfolios con Popplet, Glogster y Thinglink, así como tutoriales de manejo de estas herramientas.

Resaltamos que, se empleó Socrative para preparar un test de 100 preguntas, 45 de opción múltiple y 55 de verdadero/falso que se realizó en la sala de Informática que disponía de 25 puestos informáticos, por lo que algunos alumnos tuvieron que utilizar su propio portátil por problemas técnicos en algún ordenador.

\section{Resultados}

Para valorar el grado de satisfacción de los alumnos respecto a la materia y a la metodología utilizada, se creó un formulario de Google con 22 ítems que completaron online de manera anónima los 25 participantes. Los resultados aparecen reflejados en las Tablas 6 y 7 , y en la Figura 3.

Tabla 6: Resultados evaluación materia Eys.

\begin{tabular}{|c|l|c|c|c|}
\hline $\mathbf{N}$ & \multicolumn{1}{|c|}{ Preguntas } & SOC & PED & EyS \\
\hline 2 & $\begin{array}{l}\text { ¿A qué nivel considera que esta materia es interesante para un futuro } \\
\text { maestro? }\end{array}$ & 3,64 & 3,09 & 3,37 \\
\hline 3 & $\begin{array}{l}\text { ¿Crees que los contenidos del curso te han ayudado a comprender } \\
\text { mejor la relación entre Sociología y Pedagogía? }\end{array}$ & 2,93 & 2,93 & 2,93 \\
\hline 4 & ¿Considera que los contenidos han satisfecho sus expectativas? & 3,40 & 2,57 & 2,99 \\
\hline 5 & ¿Crees que las actividades y tareas han sido apropiadas? & 3,57 & 2,80 & 3,19 \\
\hline 6 & ¿Crees que el cronograma para las actividades ha sido apropiado? & 3,30 & 3,09 & 3,20 \\
\hline 7 & Valora la metodología desarrollada para las clases. & 3,45 & 2,55 & 3,00 \\
\hline 8 & $\begin{array}{l}\text { ¿Nos recomendaría usar la misma metodología y materiales el próximo } \\
\text { año? si }\end{array}$ & 3,9 & 2,41 & 3,11 \\
\hline 9 & Valora la evaluación de la materia utilizada por los profesores. & 3,69 & 3,25 & 3,47 \\
\hline 10 & $\begin{array}{l}\text { La evaluación y las tareas propuestas reflejaron los contenidos más } \\
\text { importantes. }\end{array}$ & 3,65 & 3,13 & 3,39 \\
\hline 11 & ¿Qué pasa con los documentos y lecturas? & 3,37 & 2,65 & 3,01 \\
\hline 12 & ¿Cuál es su opinión sobre la coordinación entre los profesores & 2,53 & 2,53 & 2,53 \\
\hline
\end{tabular}


Belo Horizonte, v. 13, n. 3, p. 161-180, set.-dez. 2020 - ISSN 1983-3652 DOI : $10.35699 / 1983-3652.2020 .25114$

\begin{tabular}{|c|l|c|c|c|}
\hline & involucrados en la materia? & & \\
\hline 13 & $\begin{array}{l}\text { ¿Considera que existen grandes diferencias entre las clases de } \\
\text { sociología y pedagogía? si }\end{array}$ & 2,81 & 2,81 & 2,81 \\
\hline 14 & El nivel de inglés del profesor es similar al de otras materias bilingües. & 3,77 & 2,05 & 2,91 \\
\hline 15 & El profesor es un experto en el tema que enseña. & 3,85 & 2,85 & 3,35 \\
\hline 16 & El profesor articula teoría y práctica. & 3,73 & 2,65 & 3,19 \\
\hline 17 & $\begin{array}{l}\text { El profesor parece estar entusiasmado con el tema y utiliza las TIC } \\
\text { correctamente. }\end{array}$ & 3,65 & 3,47 & 3,56 \\
\hline 18 & $\begin{array}{l}\text { El nivel de inglés del profesor es apropiado para enseñar esta materia } \\
\text { en el grado bilingüe. }\end{array}$ & 3,69 & 1,85 & 2,77 \\
\hline 19 & El profesor promueve la participación de los alumnos. & 3,89 & 3,77 & 3,83 \\
\hline 20 & El profesor se comunica de manera clara, fácil de entender. & 3,57 & 2,93 & 3,25 \\
\hline 21 & $\begin{array}{l}\text { El profesor ha resuelto mis dudas y preguntas correctamente en clase } 0 \\
\text { en tiempo de tutoría (departamento u online). }\end{array}$ & 3,57 & 3,05 & 3,31 \\
\hline 22 & En general, ¿cuál es su nivel de satisfacción con la materia? & 3,13 & 3,13 & 3,13 \\
\hline PRO & & $\mathbf{3 , 4 8}$ & $\mathbf{2 , 8 4}$ & $\mathbf{3 , 1 6}$ \\
\hline
\end{tabular}

Fuente: Elaboración própria.

Tabla 7: Escala de Likert de valoración materia EyS.

\begin{tabular}{|l|l|l|l|l|}
\hline \multicolumn{1}{|c|}{5} & 4 & 3 & 2 & \multicolumn{1}{c|}{1} \\
\hline Totalmente interesante & & & & Ningún interés en absoluto \\
\hline Totalmente apropiada & & & & Totalmente inadecuada \\
\hline Totalmente & & & & En absoluto \\
\hline Totalmente de acuerdo & & & & Totalmente en desacuerdo \\
\hline
\end{tabular}

Fuente: Elaboración própria.

Figura 3: Resultados evaluación materia EyS.

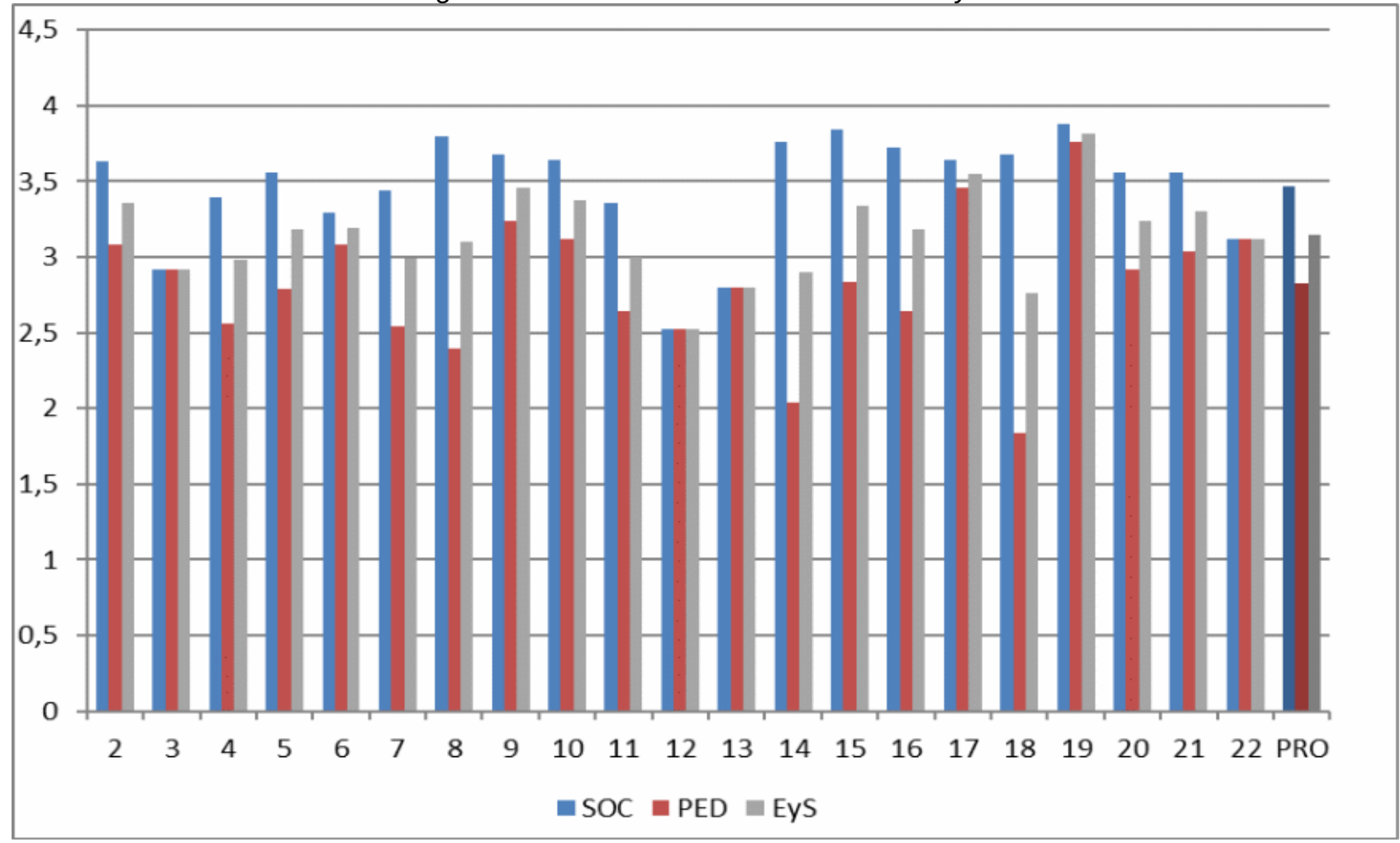

Fuente: Elaboración própria. 


\subsection{Análisis de los resultados}

Se efectuó tanto un análisis descriptivo de los datos y como un análisis inferencial, tras los cuales se puede responder afirmativamente a la pregunta que se formuló como problema fundamental de la investigación y se pudo verificar si existían diferencias de género estadísticamente significativas que permitiesen encontrar una brecha digital de género

En las Tablas 8 y 9, así como en las Figuras 4, 5 y 6, se hace un análisis descriptivo de las calificaciones obtenidas por el alumnado respecto a la variable género (GEN).

Tabla 8: Estadísticos descriptivos respecto a la variable género GEN.

\begin{tabular}{|c|c|c|c|c|c|c|}
\hline \multirow{2}{*}{} & \multicolumn{2}{|c|}{ Válido } & \multicolumn{2}{|c|}{ Perdidos } & \multicolumn{2}{c|}{ Total } \\
\cline { 2 - 7 } & $\mathrm{N}$ & Porcentaje & $\mathrm{N}$ & Porcentaje & $\mathrm{N}$ & Porcentaje \\
\hline Hombre & 1 & $89.5 \%$ & 2 & $10.5 \%$ & 19 & $100.0 \%$ \\
& 7 & & & & & \\
\hline Mujer & 4 & $93.8 \%$ & 3 & $6.3 \%$ & 48 & $100.0 \%$ \\
& 5 & & & & & \\
\hline
\end{tabular}

\begin{tabular}{|c|c|c|c|c|}
\hline \multicolumn{3}{|l|}{\begin{tabular}{|l} 
Nota_E \\
\end{tabular}} & \multirow{3}{*}{\begin{tabular}{|l|} 
Estadístico \\
8.306 \\
7.786 \\
\end{tabular}} & \multirow{3}{*}{$\begin{array}{l}\text { Error estándal } \\
.2450 \\
\end{array}$} \\
\hline \multirow{13}{*}{ Hombre } & \multicolumn{2}{|l|}{ Media } & & \\
\hline & $\begin{array}{l}\text { 95\% intervalo confianza para la } \\
\text { media }\end{array}$ & Límite inferior & & \\
\hline & & $\begin{array}{l}\text { Límite } \\
\text { superior }\end{array}$ & 8.825 & \\
\hline & \multicolumn{2}{|l|}{ Media recortada al 5\% } & 8.334 & \\
\hline & \multicolumn{2}{|l|}{ Mediana } & 8.600 & \\
\hline & \multicolumn{2}{|l|}{ Varianza } & 1.021 & \\
\hline & \multicolumn{2}{|l|}{ Desviación estándar } & 1.0102 & \\
\hline & \multicolumn{2}{|l|}{ Mínimo } & 6.3 & \\
\hline & \multicolumn{2}{|l|}{ Máximo } & 9.8 & \\
\hline & \multicolumn{2}{|l|}{ Rango } & 3.5 & \\
\hline & \multicolumn{2}{|l|}{ Rango intercuartil } & 1.3 & \\
\hline & \multirow{2}{*}{\multicolumn{2}{|c|}{$\begin{array}{l}\text { Asimetría } \\
\text { Curtosis }\end{array}$}} & -.989 & .550 \\
\hline & & & .036 & 1.063 \\
\hline \multirow{13}{*}{ Mujer } & \multicolumn{2}{|l|}{$\begin{array}{l}\text { Curtosis } \\
\text { Media }\end{array}$} & 7.998 & .1592 \\
\hline & $\begin{array}{l}\text { 95\% intervalo confianza para la } \\
\text { media }\end{array}$ & Límite inferior & 7.677 & \\
\hline & & \begin{tabular}{|l|} 
Límite \\
superior
\end{tabular} & 8.319 & \\
\hline & \multicolumn{2}{|l|}{ Media recortada al $5 \%$} & 8.046 & \\
\hline & Mediana & & 8.000 & \\
\hline & Varianza & & 1.141 & \\
\hline & \multicolumn{2}{|l|}{ Desviación estándar } & 1.0680 & \\
\hline & Mínimo & & 3.8 & \\
\hline & Máximo & & 9.9 & \\
\hline & Rango & & 6.1 & \\
\hline & \multicolumn{2}{|l|}{ Rango intercuartil } & 1.4 & \\
\hline & Asimetría & & -1.211 & .354 \\
\hline & Curtosis & & 4.077 & .695 \\
\hline
\end{tabular}

Fuente: Elaboración própria. 
Belo Horizonte, v. 13, n. 3, p. 161-180, set.-dez. 2020 - ISSN 1983-3652 DOI : $10.35699 / 1983-3652.2020 .25114$

Tabla 9: Tabla bidimensional de frecuencias respecto a la variable género (GEN).

\begin{tabular}{|c|c|c|c|c|c|c|c|c|c|c|c|}
\hline & $\begin{array}{l}\text { Nota_E } \\
=[0, \overline{1})\end{array}$ & $\begin{array}{l}\text { Nota_E } \\
=[1,2)\end{array}$ & $\begin{array}{l}\text { Nota_E } \\
=[2, \overline{3})\end{array}$ & $\begin{array}{l}\text { Nota_E } \\
=[3,4)\end{array}$ & $\begin{array}{l}\text { Nota_E } \\
=[4, \overline{5})\end{array}$ & $\begin{array}{l}\text { Nota_E } \\
=[5, \overline{6})\end{array}$ & $\begin{array}{l}\text { Nota_E } \\
=[6, \overline{7})\end{array}$ & $\begin{array}{l}\text { Nota_E } \\
=[7, \overline{8})\end{array}$ & $\begin{array}{l}\text { Nota_E } \\
=[8, \overline{9})\end{array}$ & $\begin{array}{l}\text { Nota_E } \\
=[9,10]\end{array}$ & TOTAL \\
\hline $\begin{array}{l}\text { GEN_F = } \\
\text { Hombre }\end{array}$ & 0 & 0 & 0 & 0 & 0 & 0 & 3 & 1 & 7 & 6 & 17 \\
\hline $\begin{array}{l}\text { GEN_F = } \\
\text { Mujer }\end{array}$ & 0 & 0 & 0 & 1 & 0 & 0 & 3 & 16 & 16 & 9 & 45 \\
\hline TOTALES & 0 & 0 & 0 & 1 & 0 & 0 & 6 & 17 & 23 & 15 & 62 \\
\hline
\end{tabular}

Fuente: Elaboración própria.

Figura 4: Gráfico de tendencias respecto a la variable género GEN (resultados ordenados de mayor a menor).

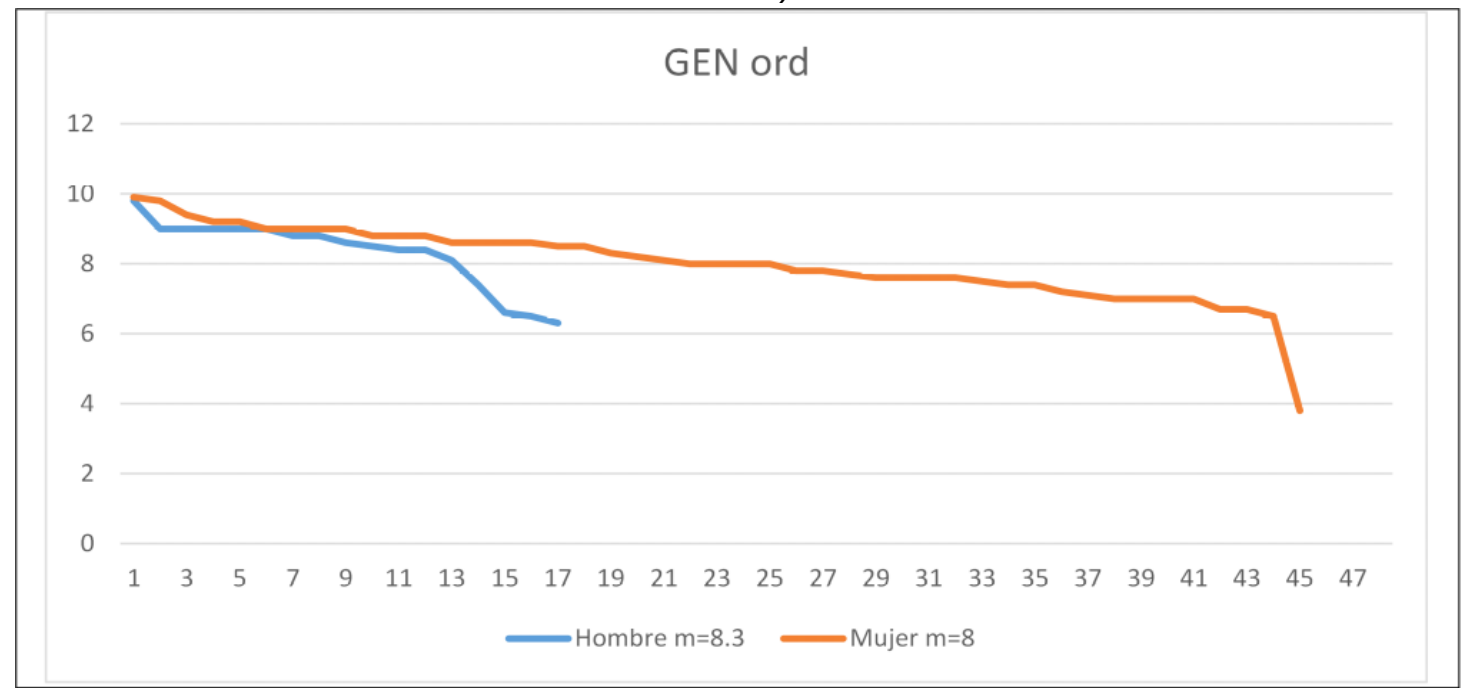

Fuente: Elaboración propia 
Figura 5: Histogramas y diagrama de interacción respecto a la variable género GEN.

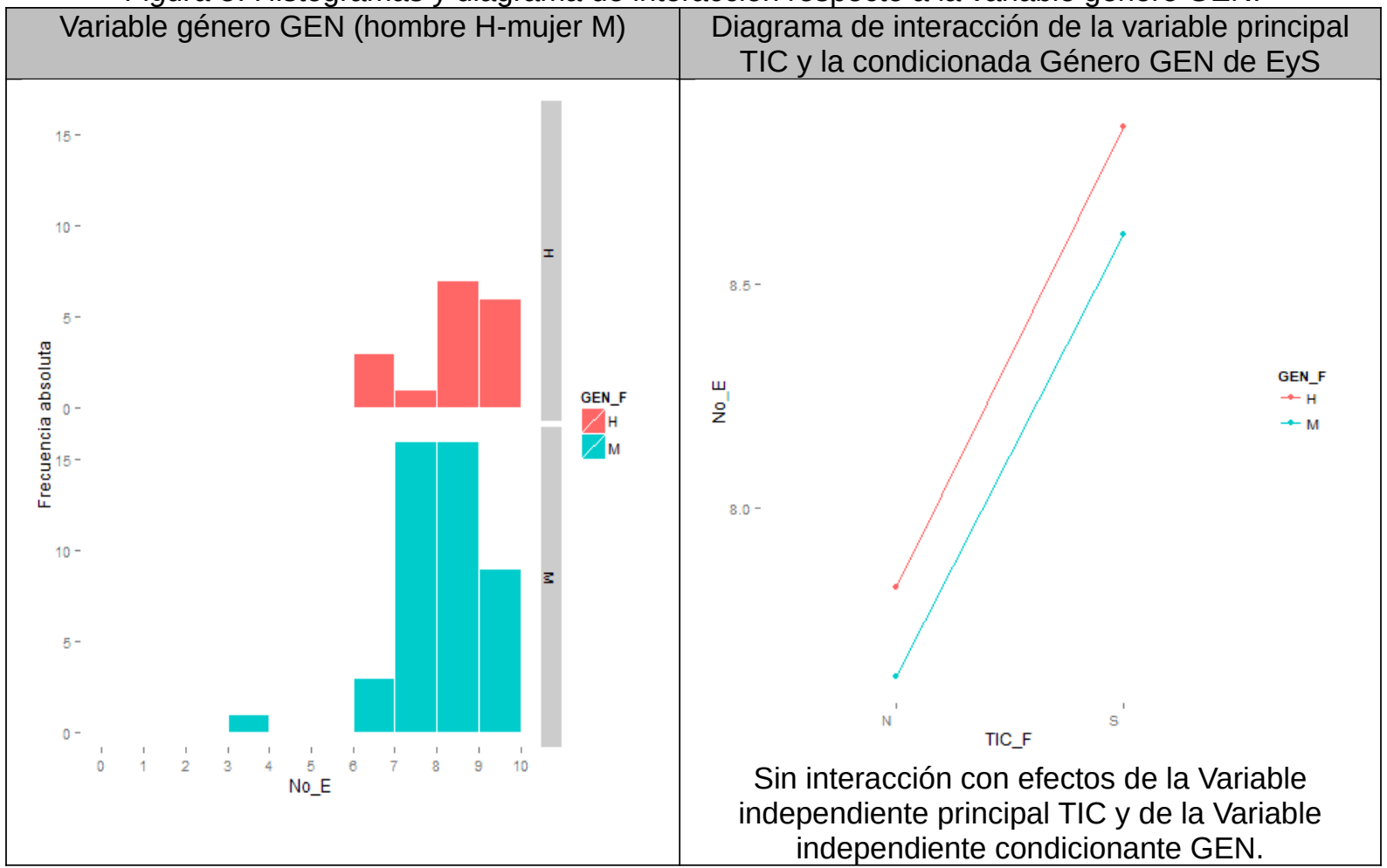

Fuente: Elaboración própria.

Figura 6: Diagramas respecto a la variable género GEN.

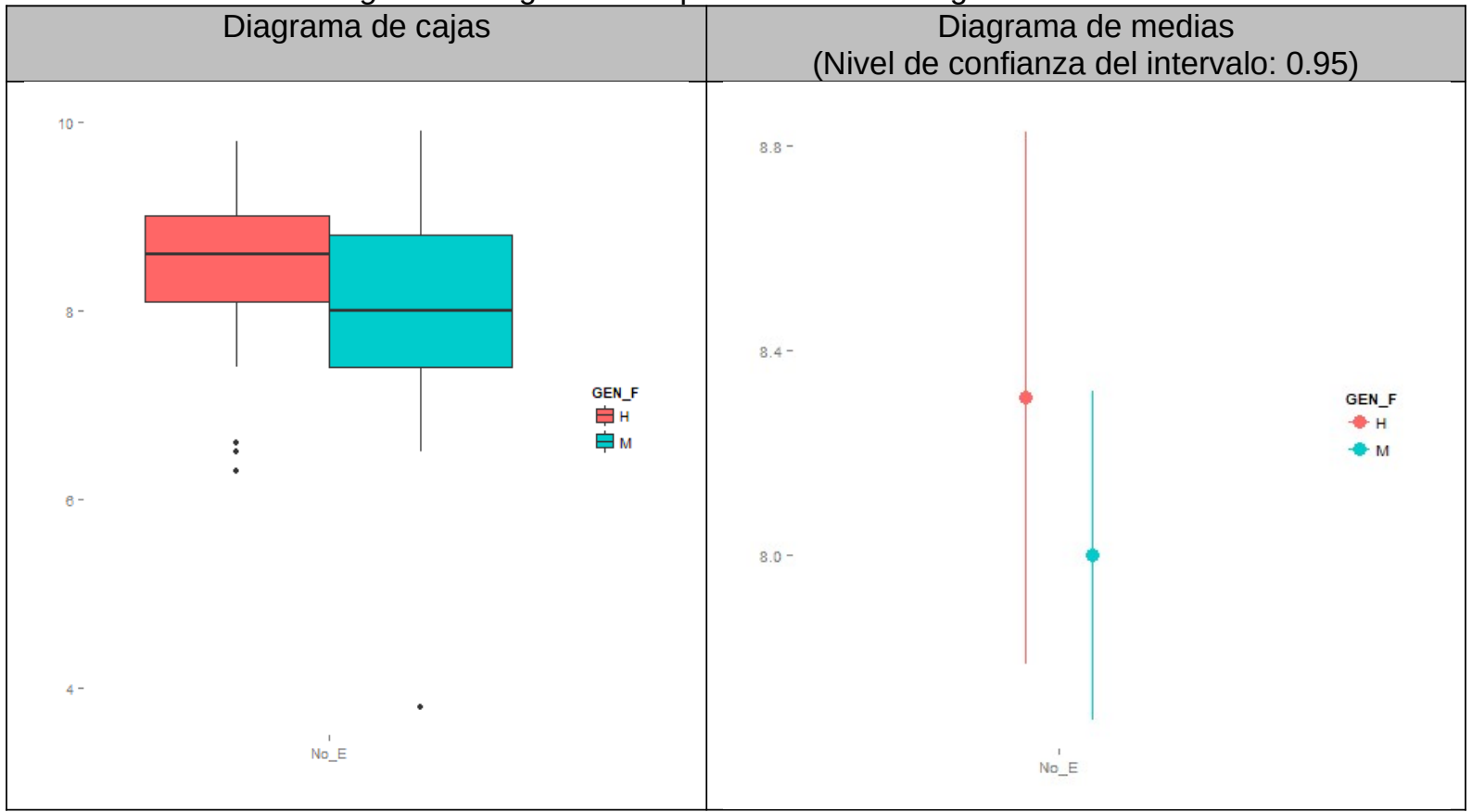

Fuente: Elaboración própria.

Analizando los datos obtenidos, podemos concluir:

- Los hombres tienen una media 308 más alta que las mujeres con una menor dispersión y una mediana .6 punto más alta que las mujeres. 
- Los coeficientes de asimetría tanto en mujeres como hombres son negativos, lo cual nos indica que en ambos géneros existe una mayor concentración de valores en la parte derecha de la media situada en la parte elevada de la escala de puntuaciones.

- La mediana de las mujeres es inferior al percentil 25 primer cuartil de los hombres y la mediana de los hombres es ligeramente inferior al percentil 75 o tercer cuartil de las mujeres.

- La curtosis o coeficiente de apuntamiento en ambos géneros son positivos, lo que nos señala que las distribuciones son leptocúrticas mostrando un alto grado de agrupación alrededor de los valores intermedios de la variable.

- Los coeficientes de asimetría son similares en ambos géneros.

- La curtosis o coeficiente de apuntamiento en los hombres es bastante menor que en las mujeres, lo que nos apunta a que los valores en los hombres están menos agrupados en la región media de la distribución que en las mujeres.

\subsection{Análisis inferencial}

Aunque el análisis descriptivo descubrió un mejor comportamiento del grupo experimental $2^{\circ} \mathrm{B}$ frente al grupo de control $2^{\circ} \mathrm{C}$. Se comprobó si esta diferencia era significativa desde un punto de vista estadístico.

Para poder responder al problema de investigación, tuvimos que verificar si podía ser rechazar la hipótesis nula. $\mathrm{HO}$ : $\mathrm{Mc}=\mathrm{Me}$, donde $\mathrm{Mc}$ y $\mathrm{Me}$ eran las medias muestrales de los grupos de control y experimental, de las calificaciones de los alumnos. Comprobamos mediante pruebas estadísticas, si podía ser rechazada la hipótesis nula; pudiendo afirmar, en ese caso, que había diferencias significativas entre los grupos que se comparaban. (BISQUERRA, 2004). Siendo necesario establecer el grado de significación, que es una representación de la probabilidad de error que se quiere asumir al rechazar la hipótesis nula. En la investigación educativa es usual asumir un nivel de significación de p del 0,05. Las técnicas inferenciales que se utilizaron fueron la prueba de normalidad de Kolmogorov-Smirnov, tanto con la corrección de significación de Lilliefors como sin ella, para comprobar la normalidad requerida para poder emplear pruebas paramétricas, también realizamos el test de Levene de comparación de varianzas que establece el estadístico a emplear en el test ANOVA de comparación de medias de muestras independientes (Ver Tabla 10).

Tabla 10: Prueba ANOVA GENTIC Modelo lineal general Univariado.

\begin{tabular}{|l|r|r|r|r|r|}
\hline \multicolumn{7}{|c|}{ Variable dependiente: } \\
\hline Origen & $\begin{array}{c}\text { Tipo III de suma de } \\
\text { cuadrados }\end{array}$ & gl & Media cuadrática & F & Sig. \\
\hline Modelo corregido & $15,945^{a}$ & 3 & 5,315 & 5,957 &, 001 \\
\hline Intersección & 3275,382 & 1 & 3275,382 & 3671,264 &, 000 \\
\hline GEN_F &, 573 & 1 &, 573 &, 643 &, 426 \\
\hline TIC_F & 12,274 & 1 & 12,274 & 13,757 &, 000 \\
\hline GEN_F* TIC_F &, 005 & 1 &, 005 &, 006 &, 940 \\
\hline Error & 51,746 & 58 &, 892 & & \\
\hline Total & 4117,710 & 62 & & & \\
\hline Total corregido & 67,690 & 61 & & & \\
\hline
\end{tabular}


$\mathrm{R}$ al cuadrado $=, 236(\mathrm{R}$ al cuadrado ajustada $=, 196)$.

Fuente: Elaboración propia.

Para la variable TIC como el nivel de significación $.000<.050$, no pudimos considerar iguales esas medias para el nivel de confianza seleccionado 95\%, por lo que tuvimos que rechazar la hipótesis nula de igualdad de medias $\mathrm{HO}$ : Mc=Me, y seleccionar la hipótesis alternativa $\mathrm{H} 1$ de diferencia de medias muestrales entre el grupo experimental y de control.

Como además, en la variable GEN el nivel de significación .426 > .050, se pudieron suponer iguales las medias para el nivel de confianza escogido $95 \%$, por lo que pudimos aceptar la hipótesis nula de igualdad de medias muestrales $\mathrm{HO}$ : $\mathrm{Mc}=\mathrm{Me}$ y rechazar la hipótesis alternativa $\mathrm{H} 1$ de diferencia de medias entre géneros en el rendimiento académico.

Resulta necesario señalar que, como para la variable intersección el nivel de significación $.000<.050$, tuvimos que aceptar que había interacción entre la variable GEN y la variable TIC. Igualmente, para la variable interacción GENTIC el nivel de significación $.940>.050$, se podía considerar iguales esas medias para el nivel de confianza elegido 95\%, por lo que se debía rechazar la hipótesis alternativa H1 de diferencia de medias y aceptar la hipótesis nula de igualdad de medias muestrales $\mathrm{HO}: \mathrm{Mc}=\mathrm{Me}$.

\section{Discusión}

El grupo experimental integrado por el grupo de clase 2B alcanzó una media de 8.69 superior a la del grupo de control 7.67, constituido por el grupo de clase $2 \mathrm{C}$, habiendo podido verificar que esta diferencia era estadísticamente significativa, lo que nos ratificó la hipótesis de la investigación.

Como se acaba de exponer, las hombres obtuvieron una media de 8.306 más alta que la de las mujeres 7.998, no siendo esta diferencia en el rendimiento académico estadísticamente significativa. Lo que nos permite afirmar, enlazando con los datos de Eurostat (2019a, 2019b), que las mujeres que son mayoría en las carreras relacionadas con la educación, en este estudio de caso tienen un rendimiento académico ligeramente inferior al de los hombres, lo que permite inferir que hemos encontrado una posible brecha digital de género en la investigación, aunque no estadísticamente significativa.

\section{Conclusión}

Se evidenció que las mujeres experimentan grandes dificultades para el manejo de las TIC de forma experta. Pese a que la primera brecha digital de género está desapareciendo, ya que se está equiparando la cifra de usuarios TIC de ambos sexos (BOOTH; GOODMAN; KIRKUP, 2010; BRYNIN, 2006; DRABOWICZ, 2014), continua estando presente dentro de la sociedad actual la segunda brecha digital (CASTAÑO, 2008; MATEOS; GÓMEZ, 2019), que viene determinada por las diferencias en las habilidades que permiten un uso experto de las TIC, lo que tiene una clara influencia en 
que sólo sean mujeres el 17\% de las alumnas de Ingeniería Informática de España (ESPEJO, 2015; MARTÍNEZ; SERNA, 2018; MATEOS; GÓMEZ, 2019; RUIZ; SÁNCHEZ, 2010).

Se valora que, el problema es consecuencia de diferentes prácticas sociales que ayudan a un menor entusiasmo de las mujeres por las tecnologías, así como del dominio masculino en las áreas donde se crean las nuevas tecnologías (BOOTH; GOODMAN; KIRKUP, 2010; CASTAÑO, 2008; GARCIA; GROS; ESCOFET, 2012; GARCÍAVALCARCER; TEJEDOR, 2017).

Como consecuencia de los resultados obtenidos, se considera necesario recomendar el establecimiento de contextos más favorables para mujeres proporcionándoles ejemplos de modelos femeninos en el ámbito de la tecnología, así como trabajar con el profesorado de institutos y colegios para plasmar acciones de divulgación en actividades informáticas (CABERO, 2006; CHAVES; TRUJILLO; LÓPEZ, 2015; LÓPEZ-NAVAJAS, 2014; MARTÍNEZ-GÓMEZ; MARÍN-GARCIA, 2015; MARTÍNEZ; SERNA, 2018; MATEOS; GÓMEZ, 2019; SÁNCHEZ, 2012).

Igualmente, sería importante destacar que, aunque el empleo de los ordenadores como herramienta de relación y comunicación es bastante parejo entre chicos y chicas, como también se ha concluido en otras investigaciones (GARCÍA-VALCARCEL; TEJEDOR, 2017; GLEDHILL, 2019; ROBERTS; FOEHR; RIDEOUT, 2005); no está sucediendo lo mismo con otros usos del ocio tecnológico, como son los videojuegos. Existiendo numerosos estudios que relacionan la brecha digital de género con los videojuegos, y con una reducción de oportunidades profesionales y educacionales para las mujeres (BEAVIS; CHARLES, 2007; JENSON; CASTELL, 2010).

Como limitación más significativa de la investigación realizada se destaca el tamaño de la muestra. Por ello, se está trabajando en otras universidades y se ha ampliado la muestra considerablemente. Sin embargo, hasta ahora, los estudios realizados no ofrecen diferencias significativas con la investigación reflejada en este artículo.

\section{Referencias}

ADELL, J.; CASTAÑEDA, L. Tecnologías emergentes, ¿pedagogías emergentes? En: HERNÁNDEZ, J; PENNESI, M; D. SOBRINO, D; VÁZQUEZ, A. (coord.). Tendencias emergentes en educación con TIC. Barcelona: Asociación Espiral, Educación y Tecnología, 2012, p. 13-32.

ADYA, M.; KAISER, K. M. Early determinants of women in the it workforce: a model of girls' career choices. Information Technology \& People, v. 18, n. 3, p. 230-259, 2005.

AREA, M.; ADELL, J. eLearning: Enseñar y aprender en espacios virtuales. En: DE PABLOS, J. (coord). Tecnología Educativa. La formación del profesorado en la era de Internet. Aljibe, Málaga, 2009, p. 391-424.

BADIA, A.; MENESES, J.; SIGALÉS, C., Percepción de los docentes sobre los factores que afectan al uso educativo de las TIC en el aula equipada de tecnología. Electronic Journal of Research in Educational Psychology, v. 11, n. 3, p. 787-808, 2013. 
BARTOLOMÉ, A.; GARCÍA-RUIZ, R.; AGUADED, I. Blended learning: panorama y perspectivas. RIED. Revista Iberoamericana de Educación a Distancia, v. 21, n. 1, p. 3356, 2018.

BEAVIS, C.; CHARLES, C. Would the 'real' girl gamer please stand up? Gender, LAN cafés and the reformulation of the 'girl' gamer. Gender and Education, v. 19, n. 6, p. 691705, 2007.

BISQUERRA, R. Metodología de la investigación educativo. Madrid: La Muralla, 2004.

BOOTH, S.; GOODMAN, S.; KIRKUP, G. Gender Differences in Learning and Working with Technology: Social Constructs and Cultural Contexts. Hershey (PA): IGI Global, 2010.

BRYNIN, M. The Neutered Computer. En: KRAUT, R; BRYNIN, M.; KIESLER, S. (ed.), Computers, phones, and the Internet. Oxford: Oxford University Press US, 2006, p. 84-96.

BURRELLI, J. Thirty-three years of women in S\&E faculty positions. National Science Foundation positions (NSF 08-308). Washington, DC: National Science Foundation. ed., 2008. Disponible en: http://www.nsf.gov/statistics/infbrief/nsf08308/nsf08308.pdf. Acceso en: 12 nov. 2019.

CABERO, J. Bases pedagógicas del e-learning. DIM: Didáctica, Innovación y Multimedia, v. 6, 2006. Disponible en: https://www.raco.cat/index.php/DIM/article/view/56479. Acceso en: 23 oct. 2019.

CARLSON, S. Wanted: female computer scientists. The Chronicle of Higher Education, v. 52, n. 19, 35-38, 2006.

CASTAÑO, C. La segunda brecha digital. Madrid: Ediciones Cátedra, 2008.

CATASÚS, M. G.; ROMEU, T.; PÉREZ-MATEO, M. Competencias TIC y trabajo en equipo en entornos virtuales. RUSC. Universities and Knowledge Society Journal, v. 4, n. 1, 2007.

CHAVES, E.; TRUJILLO, J.M.; LÓPEZ, J. Autorregulación del Aprendizaje en Entornos Personales de Aprendizaje en el Grado de Educación Primaria de la Universidad de Granada, España. Formación Universitaria, v. 8, n. 4, p. 63-76, 2015.

DRABOWICZ, T., Gender and digital usage inequality among adolescents: A comparative study of 39 countries. Computers \& Education, v. 74, p. 98-111, 2014.

ESPEJO, R.L. Interacción Simbólica en un Acto de Subjetividad de Género. Formación Universitaria, v. 8, n. 5, p. 43-58, 2015.

EUROSTAT. ICT usage in households and by individuals. Bruselas: Eurostat. 2019a. Disponible en: https://ec.europa.eu/eurostat/data/database?node code=isoc ec ibuy. Acceso en: 3 oct. 2019.

EUROSTAT. Students enrolled in tertiary education by education level, programme 
orientation, sex and field of education. Bruselas: Eurostat. 2019b. Disponible en: https://ec.europa.eu/eurostat/data/database?node_code=educ uoe enrt03":// ec.europa.eu/eurostat/data/database?node_code=educ_uoe_enrt03. Acceso en: 3 oct. 2019.

FERNÁNDEZ, F. J.; FERNÁNDEZ, M. J.; RODRÍGUEZ, J. M., El proceso de integración y uso pedagógico de las TIC en los centros educativos madrileños. Educación $X X 1, v .21, \mathrm{n}$. 2, p. 395-416, 2018.

FERNÁNDEZ, V. et al. Una aproximación a la situación de la mujer en los estudios universitarios de informática. Arbor, v. 184, n. 733, p. 877-887, 2008.

GARCÍA, F. J. Estado Actual de los Sistemas E-Learning. Teoría de la Educación. Educación y Cultura en la Sociedad de la Información, v. 6, n. 2, 2005.

GARCIA, I.; GROS, B.; ESCOFET, A. La influencia del género en la cultura digital del estudiantado universitario. Athenea Digital: revista de pensamiento e investigación social, v. 12, n. 3, p. 95-114, 2012.

GARCÍA-VALCÁRCEL, A.; TEJEDOR, F. J. Percepción de los estudiantes sobre el valor de las TIC en sus estrategias de aprendizaje y su relación con el rendimiento. Educación $X X 1,20$, n. 2, 137-159, 2017.

GIL-JUÁREZ, A.; FELIU, J.; GONZÁlEZ, A. V. Performatividad Tecnológica de Género: Explorando la Brecha Digital en el mundo del Videojuego. Quaderns de Psicologia, v. 12, n. 2, p. 209-226, 2010.

GLEDHILL, I. M. A. A global approach to the gender gap in mathematical, computing and natural sciences: How to measure it, how to reduce it? South African Journal of Science, v. 115, n. 3-4, p. 1-3, 2019.

GONZÁLEZ, A.M.; VERGÉS, N.; MARTíNEZ, J.S. Las mujeres en el mercado de trabajo de las tecnologías. Revista Española de Investigación Sociológica, v. 159, p. 73-90, 2017.

JENSON, J.; DE CASTELL, S. Gender, Simulation, and Gaming: Research review and redirections. Simulation \& Gaming, v. 41, n. 1, p. 51-71, 2010.

LÓPEZ-NAVAJAS, A. Análisis de la ausencia de las mujeres en los manuales de la ESO: una genealogía de conocimiento ocultada. Revista de Educación, v. 363, p. 282-308, 2014.

LOZANO GALERA, J. El triángulo del e-learning. AEFOL. 2004. Disponible en: http://www.aefol.com/elearning/articulos_detalle.asp?articulos=405. Acceso en: 21 nov. 2019.

MARTÍNEZ, L.; SERNA N. Disparities at the entrance door: gender gaps in elementary school. Educação e Pesquisa, v. 44, 2018. 
MARTÍNEZ-GÓMEZ, M., MARÍN-GARCÍA, J.A. Como medir y guiar cambios hacia entornos educativos más motivadores. Formación Universitaria, v. 2, n. 4, p. 63-76, 2015.

MASIE, E. Learning rants, raves, and reflections: A collection of passionate and professional perspectives. Oxford: Pfeiffer \& Co., 2005

MATEOS, S.; GÓMEZ, C. Libro Blanco de las mujeres en el ámbito tecnológico. Madrid: Secretaría de Estado para el Avance Digital. Ministerio de Economía y Empresa, 2019.

ROBERTS, D. F.; FOEHR, U. G.; RIDEOUT, V. J. Generation M: Media in the lives of 818-yearolds. Menlo Park, CA: Kaiser Family Foundation Study, 2005.

ROYSE, P. et al. Women and Games: Technologies of the Gendered Self. New Media \& Society, v. 9, n. 4, p. 555-576, 2007.

RUIZ, J.; SÁNCHEZ, J. El género como factor influyente en la estrategia para integrar las tic en la práctica docente. Pixel-bit. Revista de medios y educación, v. 37, p. 67-76, 2010.

SÁINZ, M.; LÓPEZ-SÁEZ, M. Gender differences in computer attitudes and the choice of technology-related occupations in a sample of secondary students in Spain. Computers \& Education, v. 54, n. 2, p. 578-587, 2010.

SÁNCHEZ, M. M. Diseño de recursos digitales para entornos de e-learning en la enseñanza universitaria. RIED. Revista Iberoamericana de Educación a Distancia, v. 15, n. 2, p. 53-74, 2012. 